\title{
Integración y visibilidad de la gestión de contenidos, dos retos por alcanzar en el modelo de comunicación radiofónica digital. Análisis de los casos de SER, COPE y RNE
}

\author{
Miriam RODRÍGUEZ PALLARES \\ Universidad Complutense de Madrid. Departamento de Periodismo IV \\ mrpallares@pdi.ucm.es
}

Recibido: Enero 2015

Aceptado: Marzo 2015

\begin{abstract}
Resumen: La gestión de contenidos tiene que adaptarse al nuevo modelo de comunicación digital y caminar hacia el epicentro del organigrama empresarial. En cierta medida está haciéndolo, pero los medios, por diferentes razones, bien económicas, bien por falta de perspectiva estratégica, parecen no encontrar el aliciente necesario para explotar sus posibilidades. En este texto se analiza la actividad que los departamentos de gestión de contenidos realizan en tres de las principales cadenas de radiodifusión españolas (SER, COPE y RNE) con el fin de identificar, por un lado, si se están llevando a cabo políticas vinculadas a la integración total de los fondos generados en la empresa de comunicación y, por el otro, si estos departamentos cuentan con la capacidad de crear contenidos propios con reconocimiento de autoría. Los resultados obtenidos indican que, aunque los modelos analizados son totalmente desiguales, coinciden en gestionar solamente contenidos mediáticos y tan solo el departamento de Documentación Corporativa de Cadena SER se identifica como creador y publicador de contenidos.
\end{abstract}

Palabras clave: COPE; Empresa informativa; Empresa de radiodifusión; Gestión de contenidos mediáticos; RNE; SER; Visibilidad documental.

Integration and visibility of content management, two challenges to achieve at digital broadcasting communication. Case studies about SER, COPE and RNE

\begin{abstract}
The content media management has to adapt to the new communication model and advance towards the center of the media organizational chart. Although this is currently being attempted, the different media fail to find enough motivation to exploit all the possibilities of this role, either for economic reasons or for the lack of a strategic perspective. This paper analyses the activity of the content management departments among the three main broadcasting networks in Spain (SER, COPE and RNE), with the aim of identifying practices addressed to a total integration of self-generated content and discover if these departments have the capability to generate their own content under their own signature. The results obtained indicate that all cases studied are different, but all of them manage exclusively media contents and only Cadena SER content management department writes and publishes their own contents.
\end{abstract}

Keywords: Broadcasting companies; COPE; Documentary visibility; Media companies; Media content management; RNE; SER. 


\section{INTRODUCCIÓN}

Las consecuencias que supuso la irrupción de la distribución online para los medios de comunicación tradicionales son incontables. En este escenario cambiante y ambicioso, los modelos de gestión de contenidos -documentación o variables en el significante- deben adaptarse al nuevo sistema de comunicación digital y caminar hacia el epicentro del organigrama empresarial. En cierta medida están haciéndolo, pero los medios, por diferentes razones, bien económicas, bien por falta de perspectiva estratégica, parecen no encontrar el aliciente necesario para ofrecer la confianza que precisa esta actividad empresarial.

Por un lado, este artículo se propone definir dos nuevos retos para los departamentos de gestión de contenidos radiofónicos y, por lo tanto, para sus responsables humanos: la integración trasversal de los fondos explícitos que maneja la organización, independientemente de su origen, y la creación de contenidos propios con reconocimiento de autoría.

Por otro lado, se tratará de identificar si la gestión de contenidos radiofónicos camina en la dirección marcada o si, por el contrario, se enquista en su concepción más tradicional. Para ello se presentan los resultados de un análisis descriptivo aplicado en SER, COPE y RNE, es decir, en el contexto de la empresa radiofónica, medio que los profesores Sande y Peinado describen como "una organización social que, a través de la gestión y administración de elementos humanos, técnicos, tangibles, intangibles y recursos financieros, produce, distribuye -por medio de diferentes soportes y canales- y comercializa contenidos sonoros y otros recursos multimedia que pretenden satisfacer las necesidades informativas y de entretenimiento de sus públicos objetivos, con el fin de obtener el máximo beneficio posible” (Fernández Sande y Peinado Miguel, 2012: 36).

No es casualidad que las nuevas definiciones de los medios de comunicación incluyan referencias a la condición multimedia de los mismos, multicanal diría García Lastra (2012: 169). Y es que, con mejores o peores resultados, la popularización de la comunicación digital online en el panorama comunicativo ha supuesto la adaptación de los medios a nuevos modelos de distribución y organización empresarial, una revolución en todo caso. También para la radio, ahora un medio asincrónico, fragmentado y multiformato que fomenta la comunicación bidireccional y las posibilidades del hipertexto (Cebrián Herreros, 2001; González Conde, 2010; López Vidales, 2011; González Aldea, 2011; Martínez Costa, 2011; Gallego Pérez, 2012). ¿Qué implica todo esto? La multiplicación, reutilización y vinculación de contenidos que, potenciados precisamente por las consecuencias del modelo de comunicación digital online, enriquecen la oferta radiofónica, ya sea para optimizar el servicio mediático, para autopublicitarse o para ahorrar costes. Es aquí donde tienen un papel fundamental las políticas de gestión de contenidos como salvaguarda y coadyuvante de la producción mediática. Pero, el alcance de la gestión de contenidos puede o debería 
ir mucho más allá si, como se decía al inicio, contase con un mayor respaldo empresarial que le proporcionase los recursos técnicos y humanos para lograrlo.

\section{LOS RETOS}

A continuación se describen los retos planteados para la praxis de la gestión de contenidos aplicada al entorno empresarial radiofónico, contextualizados a partir de dos bases conceptuales: la empresarial-economicista y la documental-comunicativa.

\subsection{BASE EMPRESARIAL-ECONOMICISTA}

Desde un punto de vista empresarial y en un contexto económico globalizado en el que la competitividad alcanza su máxima, las organizaciones tienen que optimizar recursos al tiempo que buscan una diferenciación a través de sus propios medios, clave para imponerse en el mercado (Rodríguez Pallares, Rodríguez Barba, Fernández Sande, 2014: 98).

La búsqueda de la optimización de recursos radiofónicos -ya sean temporales, humanos o económicos- se identifica aquí con la base economicista-empresarial. Y, cuando hablamos de la gestión de contenidos, esta optimización de recursos se vincula a la integración de fondos en un mismo repositorio de datos: la integración de fondos mediáticos y la integración de fondos empresariales.

Para hablar de la integración mediática es imprescindible echar la vista atrás y observar como, desde finales de la II Guerra Mundial y fundamentalmente a partir de 1980, los medios de comunicación han sufrido un proceso de concentración empresarial, evolucionado del individualismo a la formación de grandes grupos plurisocietarios (Pérez Serrano, 2006; Sánchez Taberero, 1991). Se buscaba, en definitiva, unir fuerzas para sobrevivir o imponerse en un contexto económico y social cambiante y ciertamente abrumador. Y, una de las principales características de este proceso de concentración es que, bajo un mismo paraguas, se aglutinan ahora diferentes marcas, tipologías mediáticas y canales de distribución con contenidos cada vez más homogéneos.

La mejora de resultados en un grupo de comunicación de estas características exige, entre otras cosas, sacar el mayor partido posible a sus contenidos como activos intangibles. Y esto ha de hacerse aprovechando las sinergias tecnológicas y organizativas, de tal modo que la empresa logre el control total de su producción y pueda reutilizarla de forma casi inmediata de acuerdo a sus intereses. Para ello, es necesario buscar la integración en todos los procesos comunes a la producción de contenidos, evitando la duplicación de tareas hasta el final de la cadena productiva, momento en el que los recursos mediáticos deberán adaptarse a los diferentes canales de distribución (García Lastra, 2012: 172). ¿Cómo lograr esta economización productiva? La respuesta es centralizando todos los recursos 
explicitados en un mismo repositorio de datos. Es aquí donde la gestión de contenidos entra en juego: su alcance funcional debe abarcar todos los procesos de producción radiofónica -es decir, todos los contenidos mediáticos o mediatizables- identificados con la misma marca o con las marcas pertenecientes a una misma empresa matriz.

Por otro lado, desde el punto de vista de la integración empresarial, se defiende aquí que el alcance funcional de la gestión de contenidos debe ir aún más allá de aquellos de carácter mediático o potencialmente mediático, debe abarcar todos los fondos generados en el entorno organizacional (producción del departamento legal, RR.HH, secretaría general...) -evidentemente reforzando la política de perfiles de acceso a esos fondos, pues no todos serán de acceso público. Así es como ocurre en otros entornos empresariales, desde las consultorías a las empresas automovilísticas, pero no es tan común en el sector de la comunicación mediática.

Es decir, el reto planteado para la gestión de contenidos es no solo la integración de fondos mediáticos, sino también de fondos empresariales de carácter transversal, siempre siguiendo un criterio de trascendentalidad informativa y organizativa, lo que se traduce en que se tendrán en cuenta todos aquellos fondos que se presupongan útiles para la empresa a corto, medio o largo plazo. El diseño estratégico y funcional para establecer esta trascendentalidad para la actividad empresarial será competencia de especialistas en las diferentes áreas funcionales de la empresa, lo que implica, incuestionablemente, un trabajo colaborativo y el posicionamiento del departamento de gestión de contenidos en una ubicación que permita su actividad transversal, evitando su dependencia de un departamento concreto.

\subsection{BASE DOCUMENTAL-COMUNICATIVA}

Para abordar esta base conceptual, se parte de la idea general de gestión de contenidos para llegar, en última instancia, al segundo reto que se plantea en esta comunicación.

Han sido muchos los teóricos que han definido el concepto de gestión de contenidos empresariales, pero en general se identifica como una actividad cuyo fin es la selección, descripción, almacenamiento, vinculación, presentación y difusión de contenidos estructurados y no estructurados y digitales útiles en un entorno organizacional, con el objeto de facilitar su reutilización a lo largo de todo su ciclo de vida (Pérez-Montoro, 2005; Eíto-Brun, 2013; Cox y Yeadon: 2002; Barceló Llauger: 2001). Esta actividad es un proceso que exige, por un lado, de un soporte tecnológico (Content Management System) y, por el otro, de habilidades profesionales especializadas (Fernández Sande, Rodríguez Barba y Rodríguez Pallares, 2012: 393) que fomentan entornos de trabajo en colaboración, vinculando a documentalistas, periodistas, técnicos o locutores, actores del "periodismo integrado” (Salaverría, Negredo y Piqué, 2008). 
En relación a esta última idea, la profesora Rubio Lacoba adelantaba, hace casi una década, que no solamente el servicio de gestión de contenidos en un medio de comunicación, sino también sus responsables humanos, habían tenido que adaptarse al periodismo digital, convirtiéndose en un perfil triangular en el que ahora confluyen habilidades documentales -fundamento profesional- y tecnológicas, pero también periodísticas (Rubio-Lacoba, 2005: 19). De hecho, existe un traspaso de competencias entre el periodista, que ha adquirido mayor autonomía en la recuperación de contenidos (Micó Sanz, Massip Massip, García Avilés, 2009: 284; Baranda del Campo: 2009: 189; Ortiz-Sobrino, 2013: 89), y el documentalista -o gestor de contenidos- que está capacitado para intensificar sus funciones creativas y enriquecer la oferta mediática y la comunicación interna.

Sin embargo, esta capacidad de creación por parte del gestor de contenidos no se explota en la medida en la que podría ni on air ni online. La comunicación digital hipertextual, se yergue como una clara oportunidad para ofrecer contenidos documentales que le aporten valor añadido al usuario. He aquí un nuevo reto para el gestor de contenidos: la creación de productos mediáticos y su publicación con reconocimiento de autoría. Esto se traduce en dar acceso al consumidor a contenidos documentales justificados, ordenados, editados y adaptados al canal de distribución.

Decía Marcos Recio que el usuario de los canales digitales está abrumado con la sobrecarga de contenidos, en un medio busca calidad basada en la investigación y respaldada por una marca. "Es posible que la información básica, la que responde a las seis $\mathrm{w}$ del periodismo clásico, esté en millones de sitios webs, casi al mismo tiempo. Pero la información de calidad, la que sustenta una información, la que la amplía, la que tiene credibilidad y aporta datos significativos solo estará en determinados medios. Especialmente, en aquellos que tengan centro de documentación” (Marcos-Recio, 2014: 132) y lo utilicen más allá de un servicio de archivo, claro.

Así, desde la base conceptual documental-comunicativa, el reto de la gestión de contenidos es precisamente colaborar con esa calidad informativa. Para ello no debe ser solo un coadyuvante en la redacción periodística, sino también un autor “aliado” del periodista (Rubio-Lacoba, 2005: 18) y, por supuesto, defender en este caso su autoría con el objeto de reivindicar el reconocimiento empresarial y social de su labor profesional.

\section{OBJETIVOS Y METODOLOGÍA}

Para realizar este estudio se ha seleccionado una muestra representativa de la empresa radiofónica generalista en España en base a los índices de audiencia del Estudio General de Medios. Así la muestra se reduce a SER, COPE y RNE. Los datos obtenidos son el resultado parcial de una investigación de mayor alcance realizada por la autora en su tesis doctoral, "Análisis de los actuales modelos de 
gestión de contenidos y conocimiento en las grandes cadenas de radiodifusión españolas: SER, Onda Cero, COPE y RNE”.

El objetivo general de esta comunicación es conocer si los actuales modelos de gestión de contenidos en la radio generalista española responden a los retos previamente planteados y, por lo tanto, están evolucionando paralelamente al modelo de comunicación digital. Así, los objetivos específicos se corresponden con cada uno de esos retos:

> OBJETIVO 1: Identificar si el modelo de gestión de contenidos de SER, COPE y RNE alcanza una integración mediática y empresarial a través del posicionamiento departamental.

> OBJETIVO 2: Identificar si los modelos de gestión de contenidos de SER, COPE y RNE contemplan y aplican la creación de contenidos mediáticos propios por parte de sus responsables y si éstos tienen visibilidad y reconocimiento de autoría de cara al oyente/usuario.

A su vez, para alcanzar estos objetivos el modelo de trabajo se fundamenta en el análisis de dos variables representativas:

> VARIABLE 1: La primera variable, vinculada al primer objetivo y por lo tanto al primer reto, es el posicionamiento organizacional de los departamentos responsables de la gestión de contenidos en la radio española. ¿Por qué tomamos esta variable como representación sintomática? Porque de su identificación se infiere el alcance funcional del departamento de gestión de contenidos, o lo que es lo mismo, a partir del estudio de su estructura organizacional podemos conocer su posición y sus responsabilidades (Echeverri, 1995: 24) en el conjunto empresarial: bien responsabilidades solamente mediáticas (integración mediática), bien responsabilidades trasversales a todos los fondos de la empresa (integración empresarial).

La ubicación del departamento de gestión de contenidos, desde una perspectiva integradora total $\mathrm{y}$ estratégica, tiene que situarse obligatoriamente en una posición que le permita nutrirse y servir de fuente a toda la actividad organizacional, su subordinación directa de un departamento demasiado específico imposibilita su aplicación trasversal. ¿Cuál es la particularidad de los medios de comunicación? El hecho de que su producción en sí misma es intangible y sujeto pasivo de gestión. Esto puede llevar a confusión, de tal manera que solo se cuide la integración mediática, obviando los fondos generados por otros departamentos que se custodian de forma descentralizada. Sin entrar de forma pormenorizada en el funcionamiento de todas las actividades de los departamentos responsables de la gestión de contenidos, por no ser el 
objeto mismo de estudio, se entiende que esta variable es representativa para alcanzar los objetivos planteados, como ejemplificación del alcance funcional de estos departamentos.

> VARIABLE 2: La variable representativa del segundo objetivo planteado es el reconocimiento de autoría de la gestión de contenidos -bien sea en formato textual o de audio (podcast)- a través de las páginas web de SER, COPE y RNE.

La visibilidad del trabajo de los gestores de contenidos en un contexto mediático ofrece dos ventajas con pretensiones diferenciadas:

- de cara al oyente/usuario, enriquece el producto informativo y ofrece garantías de calidad, ya sea on air, ya sea online.

- atendiendo a la explotación de contenidos con valor añadido, permite sacar rendimiento a contenidos archivados, es decir, comercializar -en los casos que sea posible- o afianzar a oyentes/usuarios mediante la distribución de contenidos añadidos al producto estándar con valor documental, bien sean recuperados de los fondos gestionados en el Content Management System, bien sean creados en base a esos fondos con un fin específico aniversarios, celebraciones, cronogramas, revisiones históricas de un hecho puntual...-.

Es importante destacar, en este último caso, la particularidad de los contenidos radiofónicos en términos de explotación, muchos de ellos -sobre todo los musicales- sujetos a derechos de autor, parte de lo que se denomina propiedad intelectual o "el conjunto de derechos que corresponden a los autores y a otros titulares (artistas, productores, organismos de radiodifusión...) respecto de las obras y prestaciones fruto de su creación" (Ministerio de Educación Cultura y Deporte). Toda reproducción de contenidos ajenos a la creación propia de la empresa y al margen de la emisión on air o streaming, está sujeta a un pago adicional de acuerdo a las leyes de propiedad intelectual, motivo por el cual cuando se sugiere la explotación de contenidos de archivo, se hace referencia, fundamentalmente, a contenidos de creación propia de la empresa, de naturaleza textual o sonora no musical o musical exenta de derechos de autor, en caso contrario se plantea necesario un estudio de rentabilidad. Es evidente, también, que la ausencia de la imagen como producto estrella posiciona la rentabilización de los contenidos radiofónicos por detrás de los televisivos -aunque la multicanalidad está desdibujando estas limitaciones-, sin embargo, el contenido sonoro tiene su propio nicho de interés, fundamentalmente en el mismo entorno empresarial radiofónico, pero también, aunque en menor medida, puede tenerlo fuera de él, algo que es preciso contextualizar en políticas estratégicas integrales. 
El objetivo es poner el foco de atención en esta variable como posible complemento a los actuales contenidos radiofónicos.

La línea metodológica seguida para esta investigación parte del estudio de casos múltiples, o la investigación sistematizada de diferentes realidades en base a un mismo modelo de análisis. En esta ocasión, el análisis se sustenta, mayoritariamente, en técnicas de investigación cualitativas, cuya base epistemológica según Stake es "existencial (no determinista) y constructivista” y pone el énfasis en la interpretación (Stake, 2007: 46). Así es como a partir de los datos obtenidos se llega a una serie de conclusiones interpretativas que permiten dar respuesta a los objetivos planteados.

Tabla I. Cuadro resumen modelo metodológico

\begin{tabular}{|c|c|c|}
\hline $\begin{array}{l}\text { Fases de la } \\
\text { investigación }\end{array}$ & Métodos científicos & Indicadores considerados \\
\hline $\begin{array}{l}\text { Selección de la } \\
\text { muestra de análisis }\end{array}$ & $\begin{array}{l}\text {-revisión de índices de audiencia } \\
\text { (EGM) }\end{array}$ & - oyentes/día \\
\hline Objetivo 1 & $\begin{array}{l}\text {-entrevistas en profundidad* } \\
\text {-revisión bibliográfica de } \\
\text { documentos internos }\end{array}$ & $\begin{array}{l}\text { - estructura organizativa de la } \\
\text { empresa } \\
\text { - posicionamiento organizacional y } \\
\text { dependencia orgánica del } \\
\text { departamento } \\
\text { - alcance funcional de la gestión de } \\
\text { contenidos. }\end{array}$ \\
\hline Objetivo 2 & $\begin{array}{l}\text {-entrevistas en profundidad* } \\
\text {-observación directa no } \\
\text { participante** } \\
\text {-observación de los web sites }\end{array}$ & $\begin{array}{l}\text { - identificación del documentalista } \\
\text { como autor de contenidos on air y } \\
\text { online (escrito y audio). } \\
\text {-identificación de una línea de } \\
\text { negocio o práctica habitual vinculada } \\
\text { con la comercialización o explotación } \\
\text { de los fondos documentales, con fines } \\
\text { económicos o fidelizadores. }\end{array}$ \\
\hline
\end{tabular}

Fuente: elaboración propia.

La información se ha obtenido además de las entrevistas personales con:

AFUERA, Ángeles (abril 2012-abril 2013). Jefa del departamento de Documentación Corporativa de Cadena SER.

SÁNCHEZ, Alicia (julio 2010). Documentalista de Cadena SER Madrid.

PÉREZ, Mónica Belén (mayo 2012-abril). Directora de la subdirección de Fondos Documentales de RNE.

SÁNCHEZ, Maribel (julio 2012). Antigua responsable y creadora del Departamento de Documentación de Cadena COPE.

RODRÍGUEZ-PI, Luis (agosto 2012). Director de Antena de Cadena SER Madrid. 
AYALA, Alicia (agosto 2012). Documentalista de Cadena COPE.

MARTÍN PÉREZ, Luis (abril 2013). Jefe del área Internet de Cadena COPE.

TELLECHEA, José Manuel (abril, 2013). Director de RNE Huelva.

PINHEIRO, Luis (septiembre, 2013). Subdirector de Proyectos de RTVE.es

\section{ANÁLISIS DE CASOS}

De forma coherente a lo antedicho, el análisis de los resultados obtenidos se estructura de acuerdo a las siguientes variables/ítems:

$>$ Base empresarial-economicista: Posicionamiento organizacional.

$>$ Estructura organizativo de la empresa.

$>$ Posicionamiento organizacional y dependencia orgánica del departamento.

$>$ Alcance funcional de la gestión de contenidos.

$>$ Base documental comunicativa: autoría y visibilidad del departamento.

> Creación de contenidos propios por parte del departamento de gestión de contenidos.

$>$ Visibilidad de contenidos documentales y reconocimiento de autoría de cara al oyente/usuario.

$>$ Explotación de contenidos de creación propia con fines lucrativos o fidelizadores.

\subsection{CADENA SER}

\subsubsection{BASE EMPRESARIAL-ECONOMICISTA: POSICIONAMIENTO ORGANIZACIONAL}

Prisa Radio atiende a un modelo organizativo divisional típico de multinacionales o grupos empresariales y que "implica una subdivisión parcial de la empresa general, creando una serie de divisiones, establecidas según criterios diversos, que desarrollan por sí mismas buena parte de las funciones empresariales” (Mateo, Bergés, Sabater, 2009: 201). En este caso, las divisiones responden fundamentalmente a marcas y mercados geográficos, fruto de las políticas de expansión y adquisición por parte del Grupo Prisa que, como conglomerado empresarial enfocado a la educación, información y entretenimiento basa, además, sus divisiones en productos o formatos.

Cadena SER cuenta con un servicio de documentación mediático autónomo y reconocido dese 1988, momento en el pasa a formar parte del accionariado de Prisa Radio. En el antiguo departamento de Gestión de Contenidos de la sede central de Prisa Radio en Madrid, el organigrama planteado se adaptaba, hasta 2010 (fecha en la que se realiza una reorganización empresarial), a la estructura 
del modelo clásico lineal-staff (Mateo, Bergés, Sabater, 2009: 197). Este modelo es característico de empresas con cierta complejidad tecnológica, productiva, financiera y fiscal. Una de las particularidades de este modelo es delegar parte de las funciones de los directivos en un asistente con función consultiva, pero sin autoridad directa sobre los puestos subordinados, preservando, de este modo, la línea jerárquica única.

Figura 1. Organigrama del departamento de Gestión de Contenidos de Cadena SER (hasta 2010).

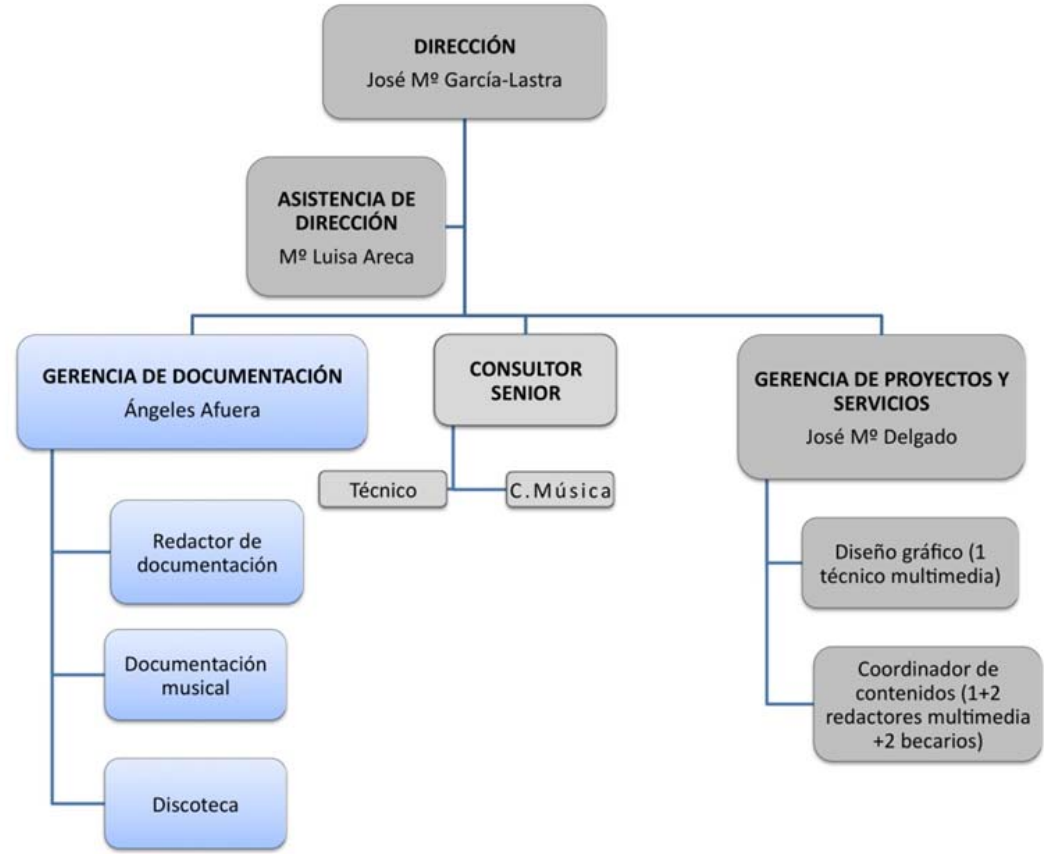

Fuente: antiguo departamento de Gestión de Contenidos y elaboración propia.

El departamento de Gestión de Contenidos fue disuelto en julio de 2010. En su ausencia se creó el departamento de Documentación Corporativa, dependiente de la dirección general de Prisa Radio y ahora cabecera documental de todo el grupo radiofónico, es decir, en él se centralizan los contenidos mediáticos o mediatizables vinculados a las diferentes marcas radiofónicas, generalistas y temáticas, pertenecientes a la matriz, Prisa Radio. El grupo Prisa Radio tiene operatividad y especial relevancia en el ámbito latinoamericano a través de diferentes marcas radiofónicas que, en su totalidad, pueden consultar las bases de datos del sistema de gestión de contenidos del grupo, Enciclomedi@, pero tan solo cuatro países, junto con España, cuentan con la capacidad de nutrir al sistema de gestión de contenidos digital, éstos son: México, Argentina, Chile y Colombia, cuya actividad gestora de contenidos depende organizativamente de su empresa nacional (W Radio, Continental, ADN y Radio Caracol), pero profesionalmente de 
la sede documental de Madrid, cabecera del modelo de gestión de contenidos digitales. Con la reformulación antes planteada, se suprimieron las figuras consultivas en el organigrama de la sede madrileña, órgano coordinador y decisor de la actividad documental, asemejando el modelo de organización al clásico lineal-jerárquico con las variables propias de un entramado empresarial en red tan amplio como el que forma el Prisa Radio en su conjunto.

El cambio en la nomenclatura y alcance del departamento representó también cambios organizativos en cuanto a sus divisiones funcionales y a sus competencias. Las funciones propias del antiguo departamento de Gestión de Contenidos se disocian ahora en dos áreas departamentales vinculadas, pero con competencias y dependencias propias: Documentación Corporativa, y Organización y Sistemas, departamento al que pasan las competencias de la antigua área de Proyectos y Servicios (en el cuadro anterior se muestran en gris las divisiones desaparecidas).

El organigrama de la plantilla en el primer cuatrimestre de 2013 responde al siguiente dibujo:

Figura 2. Organigrama del departamento de Documentación Corporativa.

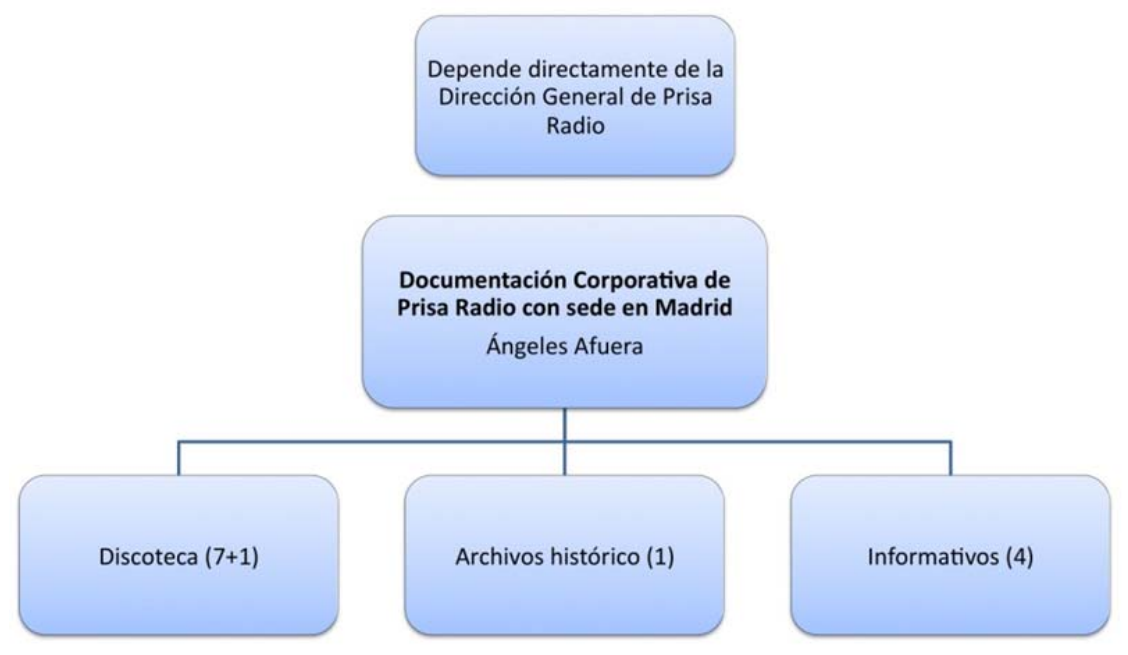

Fuente: elaboración propia.

El modelo de trabajo de Cadena SER es particular al pertenecer, como se decía anteriormente, a un grupo radiofónico de cobertura internacional. Su modelo de gestión de contenidos es la cabecera de Prisa Radio, de él depende la coordinación y la toma de decisiones transversales vinculadas a la selección, evaluación, descripción o permisos de acceso de toda la red radiofónica española y americana.

Si atendemos a la perspectiva mediática, el modelo de Cadena SER cumple el objetivo de evitar la duplicación de trabajo en el proceso de producción al integrar 
en sus competencias los contenidos generados en todas las marcas pertenecientes a Prisa Radio, no así a todas los medios del Grupo Prisa.

Su posicionamiento orgánico depende directamente de la dirección general de Prisa Radio, de esto se colige su autonomía. Cuenta con el respaldo empresarial apropiado, pero, desde la perspectiva integradora, su alcance no trasciende más allá de los contenidos mediáticos o mediatizables.

\subsubsection{BASE DOCUMENTAL-COMUNICATIVA: AUTORÍA Y VISIBILIDAD DEL DEPARTAMENTO}

De forma externa, el departamento de Documentación Corporativa encuentra visibilidad directa en antena y en el portal online a través de publicaciones propias con reconocimiento de autoría.

A lo largo de 2012 el Departamento de Documentación Corporativa amplió sus competencias para asumir procesos editoriales secundarios, es decir, además de contenedor de contenidos, se ha convertido en plataforma de publicación web, produciendo contenidos susceptibles de divulgación sin la redacción de un periodista. Para ello, se reforzó la vinculación de las herramientas de gestión de contenidos con las líneas de negocio online, así se crearon pasarelas automáticas de publicación desde la herramienta de gestión de contenidos, Enciclomedi@, y las webs corporativas. Esto se traduce en un avance cualitativo desconocido hasta el momento que huye del concepto de archivo estático para apostar por una plataforma de distribución de contenidos dinámica, también hacia dispositivos móviles.

Figura 3. Podcast en cadenaser.com, ejemplo del reconocimiento de autoría documental.

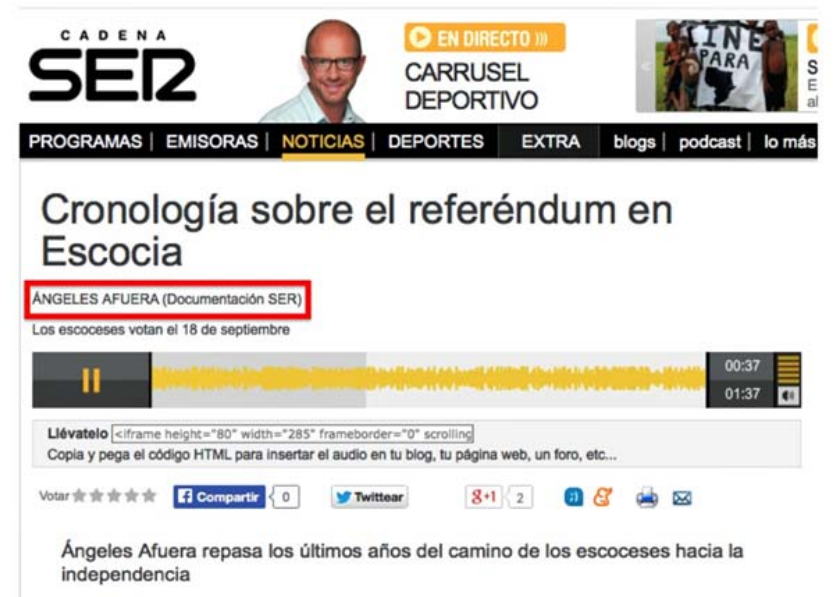

Fuente: www.cadenaser.com [consulta: 24/10/14] 
En este caso, es posible escuchar la voz del documentalista on air o leer su nombre a veces acompañado del departamento al que pertenece en el web site de la marca.

No es de extrañar que sea la firma de Ángeles Afuera la más reconocible; se puede considerar, salvando las distancias, una documentalista estrella, como tantos ejemplos profesionales pueden identificarse en el panorama periodístico y radiofónico de nuestro país.

Este reconocimiento da visibilidad a la labor documental tanto dentro de la empresa como por parte de los oyentes. Estratégicamente hablando, demuestra un trabajo contextual visible y refuerza el valor de marca.

Figura 4. Podcast en cadenaser.com ejemplo del reconocimiento de autoría documental en un especial por el aniversario de la primera emisión radiofónica en España.

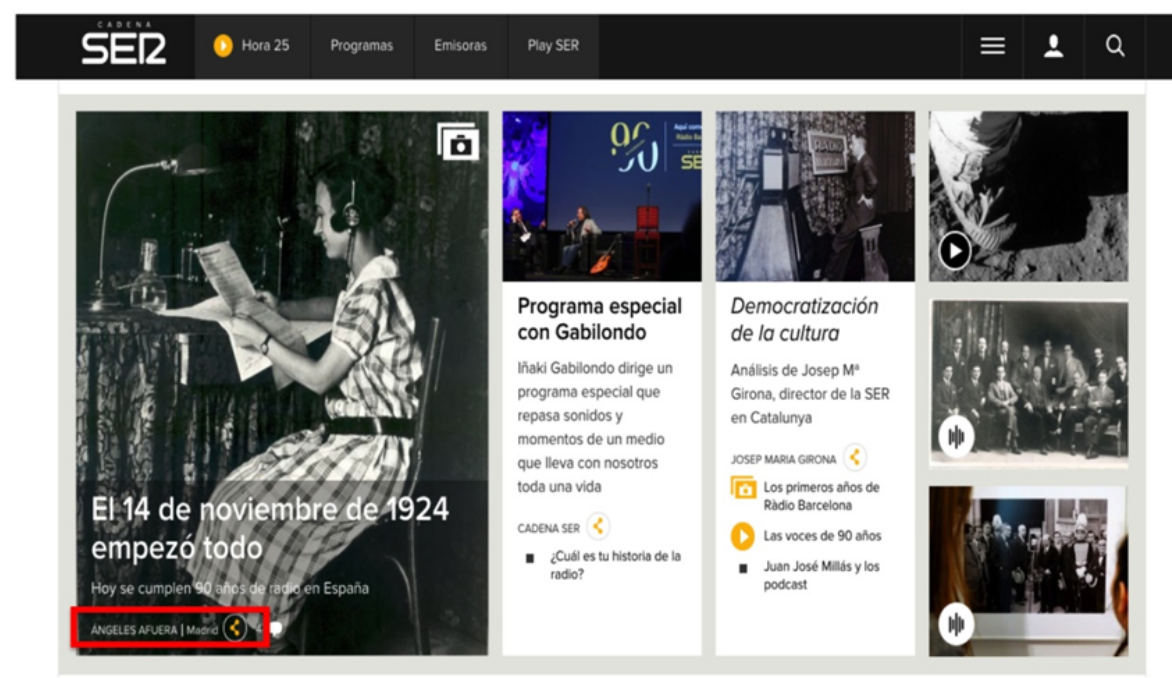

Fuente: www.cadenaser.com [consulta: 14/11/14]

En cuanto a la explotación de contenidos, el modelo de Prisa Radio ha visto reducidas sus estrategias de fidelización y publicidad de su actividad. La antigua gerencia de Proyectos y Servicios era la responsable de gestionar las políticas de rentabilización de contenidos a través de su venta o distribución de productos basados en contenidos de creación propia. La reubicación de este departamento y las reducciones presupuestarias han aletargado esta labor de forma muy notoria hasta el punto de que esta línea de negocio ya no cuenta con proyecciones a largo plazo como parecía prometer. 


\subsection{CADENA COPE}

\subsubsection{BASE EMPRESARIAL-ECONOMICISTA: POSICIONAMIENTO ORGANIZACIONAL}

El departamento de Documentación de la cadena COPE existe de forma reconocida desde 1989, paralelamente a un proceso de reajustes de la emisora en Madrid que, a nivel cualitativo, supuso la concienciación del capital intangible de la empresa como valor de uso colectivo y no solamente probatorio.

Radio Popular como grupo empresarial responde a un modelo divisional también fiel al criterio de respeto a las marcas, a los productos y a la división organizativa geográfica nacional. Si nos ceñimos solamente al modelo de gestión de contenidos, el sistema se centraliza en Madrid. También en este caso, se ciñe su alcance funcional a contenidos mediáticos o susceptibles de serlo, de acuerdo a un criterio de trascendencia informativa nacional y relegando. Los contenidos de discoteca, son relegados a un segundo plano en esta ocasión, su tratamiento, descripción y complementación informativa es escaso, así que la actividad del departamento se focaliza en conservar el continuo de las emisiones y contenidos seleccionados previamente por los redactores.

Radio Popular cuenta con un departamento documental mucho más pequeño que el de sus análogos en este estudio, con una estructura organizacional semejante al modelo lineal-jerárquico. El servicio está formado por dos figuras fijas (en el primer trimestre de 2013) y rotación de becarios, cuyo número fluctúa en función de la época desde dos a ninguno. Se ubica bajo la dependencia directa de la jefatura de Programas, a su vez subordinada de la dirección general, aunque nutre de contenidos a todas las divisiones informativas de la cadena que lo soliciten.

El caso de la COPE es el ejemplo de la subordinación de un departamento de documentación a una dirección demasiado específica. Su posicionamiento organizacional limita su autonomía, reduce su visibilidad empresarial y es incoherente con la transversalidad y la integración de contenidos defendida en la teoría.

Gráficamente podría resumirse, de forma muy simplificada, del siguiente modo: 
Figura 5. Ubicación del departamento de Documentación en COPE.

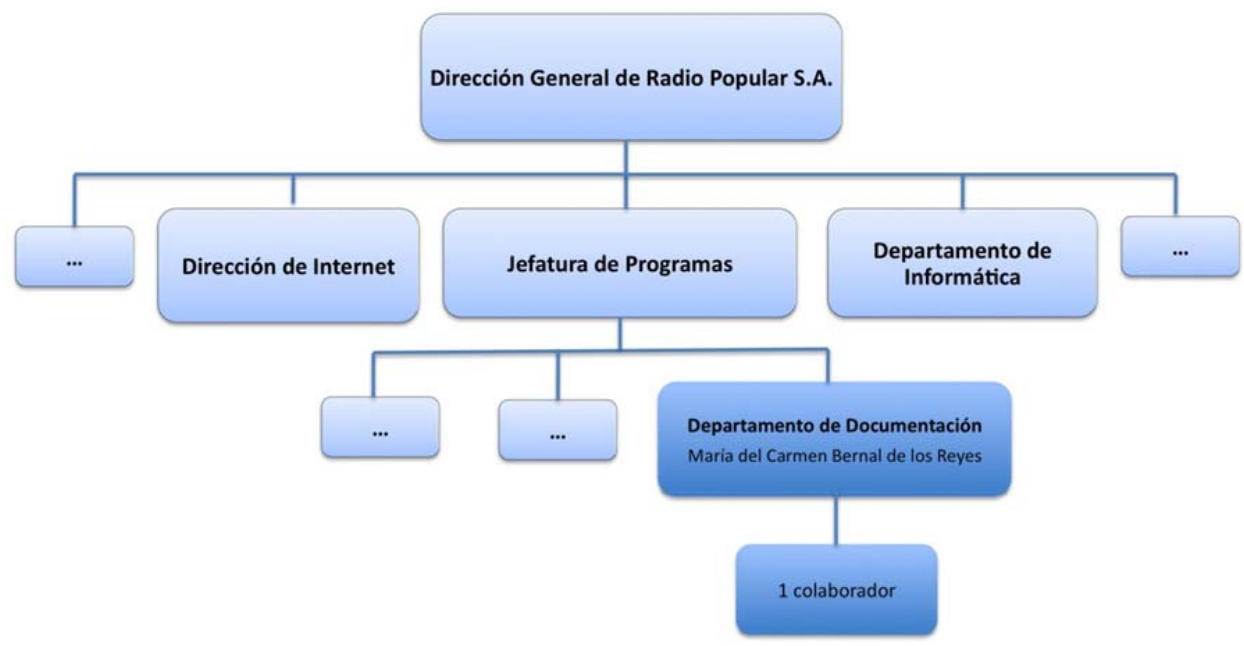

Fuente: elaboración propia.

\subsubsection{BASE DOCUMENTAL-COMUNICATIVA: AUTORÍA Y VISIBILIDAD DEL DEPARTAMENTO}

De forma externa y de cara al oyente/usuario, la visibilidad del departamento de COPE es inexistente. Aunque el equipo documental sí crea contenidos en base a criterios de previsión informativa -como suele ser habitual en estos departamentos-, éstos no se emiten ni se cuelgan online sin el barniz de un periodista. Es decir, los documentalistas no generan contenidos publicables y en ningún caso se reconoce de forma externa su colaboración con la creación de las piezas.

Por su parte, la explotación de contenidos es muy puntual "porque no hay mucha demanda" (Sánchez Redondo, julio 2012) y suele reducirse a fondos vinculados a sus particularidades como marca, respondiendo a su target objetivo. Uno de los ejemplos más ilustrativos fue la comercialización de los discursos del papa Juan Pablo II.

\subsection{RADIO NACIONAL DE ESPAÑA (RNE)}

\subsubsection{BASE EMPRESARIAL-ECONOMICISTA: POSICIONAMIENTO ORGANIZACIONAL}

Contextualizar la división funcional de RNE implica, obligatoriamente, a la Corporación de RTVE S.A. que atiende a un modelo divisional por productos mediáticos: televisión, radio y medios interactivos -atienden a los portales web de televisión y radio- y, al mismo tiempo, por funciones que dependen directamente 
del Gabinete del Presidente: recursos humanos, sistemas informáticos, fondos documentales... En un segundo nivel funcional, se presenta una división geográfica que atiende a las coberturas territoriales nacionales e internacionales.

Centrándonos exclusivamente en RNE, la organización sigue un modelo divisional por funciones que luego subdividen sus competencias por marcas RNE, Radio Clásica, Radio3, Radio4 y Radio5, Radio Exterior- y mercados geográficos -local, nacional o internacional-. Así, de la dirección de RNE, dependen tres grandes direcciones y una subdirección.

Figura 6. Ubicación de la subdirección de Fondos Documentales en el organigrama de RNE.

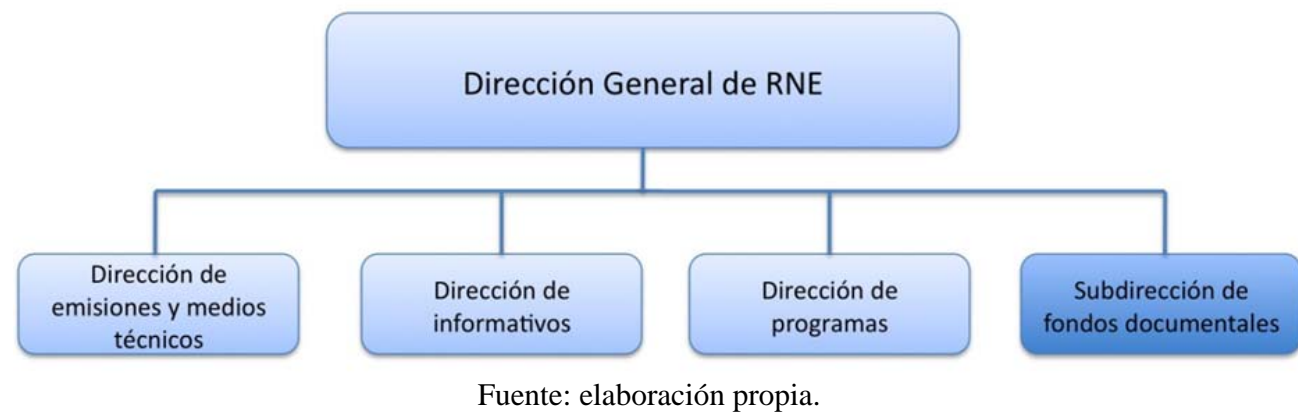

La diferencia entre dirección y subdirección se justifica con la magnitud de su plantilla, no con la importancia de su función, ni con su posicionamiento en la jerarquía empresarial. Las 43 personas adscritas a la subdirección de Fondos Documentales de RNE (SFD de RNE) pueden significar tan solo la mitad de la plantilla de algunas de sus direcciones hermanas.

La SFD de RNE depende directamente de la dirección general de RNE, pero al mismo tiempo es coordinada por un mando intermedio: la dirección de Fondos Documentales Corporativa, cabeza de la cual dependen también la SFD de Televisión y SFD de Informativos de Televisión. Aun cuando las tres subdirecciones trabajan de forma coordinada, atienden a una división orgánica por productos.

Por lo tanto, bajo un mismo órgano coordinador se gestionan contenidos radiofónicos y televisivos, es el único ejemplo cuyo alcance documental abarca marcas de tipologías mediáticas distintas. 
Figura 7. Dirección de Fondos Documentales de la Corporación de RTVE y desglose organizativo de la subdirección de Fondos Documentales de RNE.

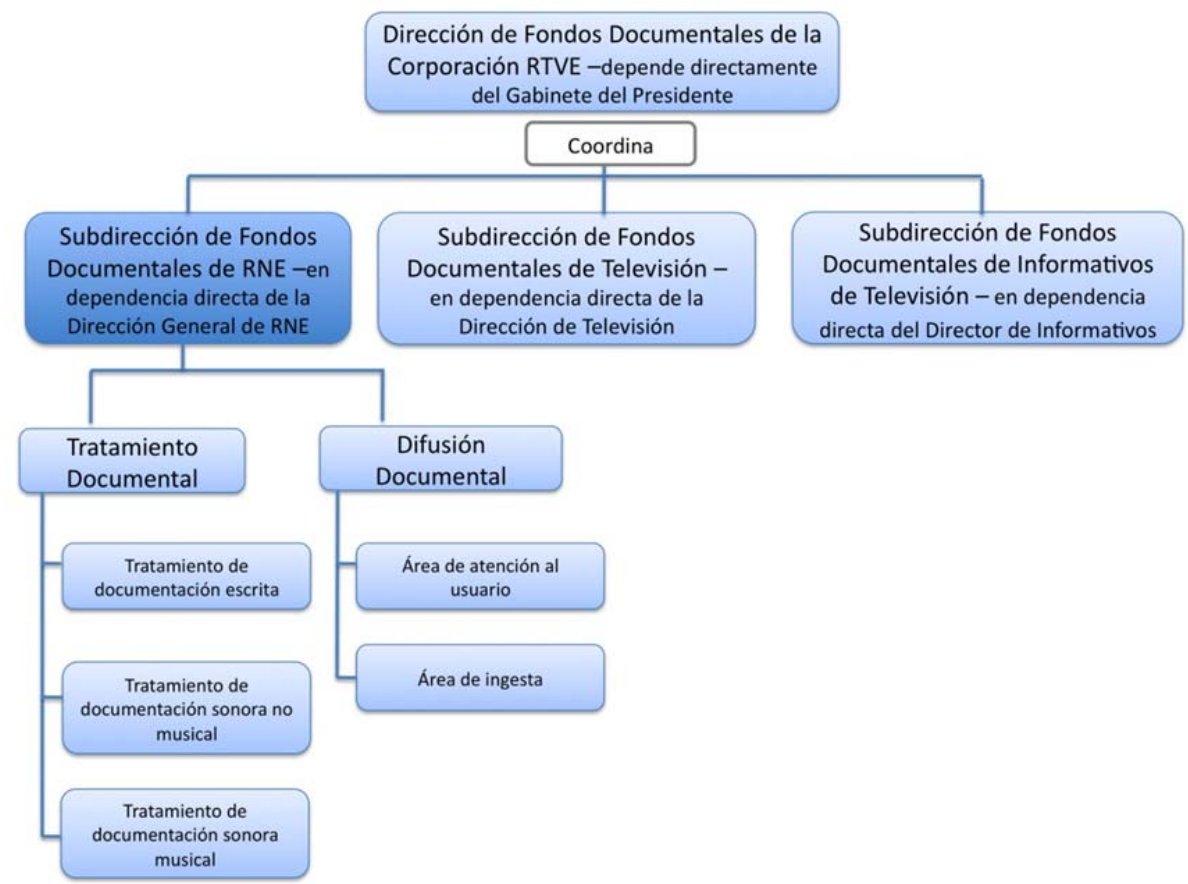

Fuente: elaboración propia.

De forma interna, la SFD de RNE se divide en dos áreas bien diferenciadas que se reflejan en el cuadro anterior:

- Tratamiento Documental. Subdividida, a su vez, en tres categorías: tratamiento de documentación escrita, tratamiento de documentación sonora no musical y tratamiento de documentación sonora musical;

- Difusión documental. Subdividida en dos áreas: área de atención al usuario y área de ingesta.

Su posicionamiento organizacional, al igual que en el caso de SER, cuenta con el reconocimiento empresarial a su actividad autónoma, pero su alcance funcional, como en SER y COPE, no llega más allá de la producción mediática o contenidos mediatizables.

\subsubsection{BASE DOCUMENTAL-COMUNICATIVA: AUTORÍA Y VISIBILIDAD DEL DEPARTAMENTO}

De cara al oyente/usuario el trabajo documental en RNE no cuenta con una visibilidad importante: los documentalistas no tienen acceso al micrófono y su autoría no es reconocida en ningún caso, algo que no hace sino mantener viva la 
naturaleza secundaria del trabajo documental. A continuación se presenta una imagen de un producto con visibilidad online creado ex profeso por el equipo de trabajo de la SFD de RNE con motivo del $75^{\circ}$ aniversario de la radio pública, pero cuya autoría no ha sido reconocida de forma visible para el usuario. Además, no se cuenta con pasarelas de publicación directas desde la subdirección de Fondos Documentales de RNE y la web.

Figura 8. Ejemplo de un trabajo creado por la subdirección de Fondos Documentales de RNE, con visibilidad online, pero sin reconocimiento de autoría.

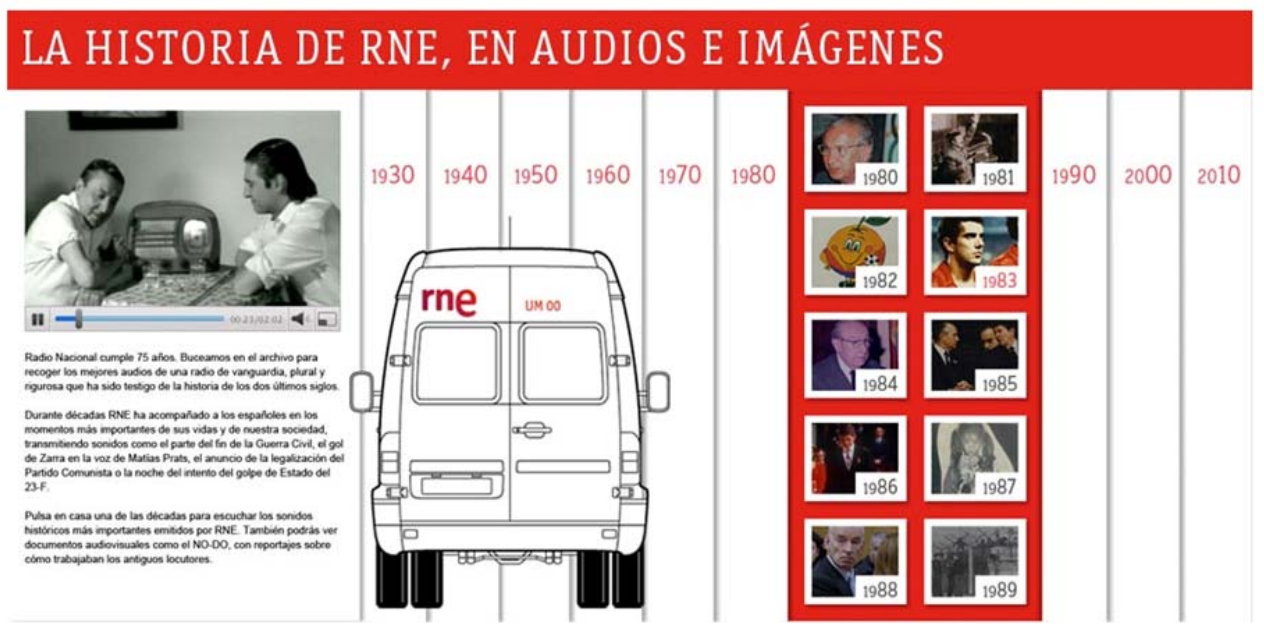

Fuente: www.rtve.es [consulta: 18/03/13]

En el caso de la radio pública, el canal de salida externo está cerrado a particulares, y la explotación se traduce en cesión de contenidos para coproducciones de RTVE.

\section{DISCUSIÓN Y CONCLUSIONES}

La principal conclusión a la que se llega tras el análisis de los datos, es que los ejemplos analizados se perfilan de modo totalmente desigual, aunque se reconoce una infraexplotación del servicio de gestión de contenidos en todos ellos.

Desde la óptica empresarial-economicista, ninguno de los casos objeto de este estudio cuenta con un departamento de gestión de contenidos que opere de modo integracionista, es decir, que centralice contenidos generados en diferentes áreas del entorno empresarial. Solo se identifica la actividad documental dedicada al fin específico de informar, formar y entretener, así es que solo se gestiona la producción mediática. 
Atendiendo a la variable considerada para este caso, se identifican dos modelos de dependencia jerárquica: dependencia directa de la presidencia o dirección de la empresa (SER y RNE) y dependencia directa de la dirección de Programas (COPE). La ubicación de los departamentos responsables de la gestión de contenidos en SER y RNE se sitúan organizacionalmente en una posición en la que podrían asumir nuevas competencias ante la implantación estratégica de políticas integracionistas empresariales, es decir, podrían asumir la gestión de contenidos más allá de la producción mediática. Esta ubicación puede entenderse como una declaración de intenciones o simplemente interpretarse como el reconocimiento organizacional de la gestión de su producción mediática. En el caso de COPE, el alcance de este departamento se reduce desde la misma jerarquía empresarial: la gestión de contenidos se considera subordinada a la producción mediática en lugar de cooperante de la misma y se obvia, por tanto, su autonomía en el organigrama empresarial.

Por su parte, desde la base conceptual documental-comunicadora, tan solo el departamento de Documentación Corporativa de Cadena SER se define como publicador de contenidos web y desarrolla una labor de creación y publicación reconocida a nivel organizacional, con visibilidad en la versión online y en la versión hertziana. En el caso de RNE, tan solo tienen visibilidad, sin reconocimiento de autoría, producciones propias de la SFD en casos esporádicos, justificados documentalmente hablando. Y, en COPE, la actividad del departamento analizado queda relegada, por el momento, a la puntual colaboración con los redactores, nunca genera contenidos propios de forma autónoma y por supuesto no disfruta del reconocimiento de autoría de cara al oyente/usuario. En este sentido, la aportación documental a la programación de Cadena SER es la más visible entre los casos analizados, hecho que distingue este ejemplo de los demás y repercute directamente en el producto, en la plantilla y en la profesión.

De acuerdo al segundo punto a tener en cuenta desde la base conceptual documental-comunicadora, la explotación de contenidos con fines fidelizadores o lucrativos apenas es considerada. El modelo de Cadena SER, que en su momento sí destinó recursos para distribuir o comercializar contenidos, ha menguado su actividad al respecto, como también ha reducido la edición de publicaciones especiales con valor añadido y fin fidelizador, esta reacción es justificada en términos económicos. En COPE se reconocen puntuales casos de distribución de contenidos documentales, pero no existe estrategia comercial a este respecto. Diferente es el caso de RNE que, sin comercializar, sí cede contenidos de archivo como norma a modo de trueque en coproducciones de la Corporación RTVE, fundamentalmente películas o series de televisión, así que de acuerdo a esta situación sí sacan partido a sus fondos en la medida en que su condición empresarial se lo permite.

Recuperando la idea inicial planteada en este punto, la heterogeneidad de los modelos analizados encuentra su punto de afinidad en la carencia de una 
perspectiva estratégica que potencie la explotación de la gestión de contenidos, como integrador de toda producción explicitada de la organización y como creador de contenidos propios.

Queda un largo camino por recorrer que exige investigaciones más profundas, así es que, en esta ocasión, se proponen, de forma sintética, las siguientes líneas de actuación, con la intención de plantear una reflexión a partir de un análisis sintomático:

> creación de un sistema de gestión documental centralizado que incluya en un mismo contenedor de contenidos aquellos generados en las líneas de negocio vinculadas a la comunicación mediática propiamente dicha y aquellos generados en otras líneas de negocio de la empresa radiofónica como RR.HH., área comercial y marketing, área legal o de asesoramiento jurídico, redacción o departamento financiero, siguiendo los criterios de trascendentalidad informativa $\mathrm{y}$ organizativa, como se menciona anteriormente. Esta propuesta contempla que cada línea de negocio cuente con aplicaciones informáticas de gestión propias, vinculadas a su vez a este repositorio central $\mathrm{y}$ en las cuales se introduzcan metadatos descriptivos básicos; una vez en el repositorio central, custodia de toda la actividad organizativa, será donde se realice el proceso documental pormenorizado. Como ya se ha mencionado, no todos los fondos pueden estar disponibles para cualquier trabajador de la empresa de acuerdo al criterio de confidencialidad, con lo cual, se definirán perfiles de acceso que, en función de la categoría profesional del demandante, posibilitarán o no el acceso a unos contenidos u otros.

$>$ concesión de nuevas responsabilidades para el perfil del gestor de contenidos/documentalista, en concreto, la capacidad para generar contenidos propios con visibilidad de autoría que complementen la oferta mediática radiofónica, bien en la web, bien on air, por ejemplo, cronologías o compilaciones históricas sobre un determinado hecho, en texto o archivo sonoro. Esta propuesta contempla la capacidad de redacción o locución por parte del gestor de contenidos/documentalista, lo hace de acuerdo a los cambios en los perfiles profesionales sucedidos como consecuencia de la multicanalidad del nuevo modelo de difusión de contenidos mediáticos (Sánchez García, et. al., 2015; López Vidales y Ortiz Sobrino, 2011; García Lastra, 2012), se plantea, por tanto, la colaboración entre periodistas y documentalistas en términos de acceso a las bases de datos y en términos redaccionales y de creación de contenidos (Gauchi-Risso, 2012). 


\section{BIBLIOGRAFÍA}

AIMC (2014). “Resumen general EGM- octubre 2013 a mayo 2014”. Disponible en: www.aimc.es/-Datos-EGM-Resumen-General-.html. [Consulta: 31/10/14]

BARANDA DEL CAMPO, Cristina (2012). "La barrera entre el periodista y el documentalista”. Documentación de las Ciencias de la Información, vol.35, pp. 101-117 http://dx.doi.org/10.5209/rev_DCIN.2012.v35.40448

BARCELÓ LLAUGER, María (2001). Hacia una economía del conocimiento. Madrid: ESIC.

CADENA SER (2014). Disponible en: www.cadenaser.com >. [Consulta: 24/10/14]

CEBRIÁN HERREROS, Mariano (2001). La radio en la convergencia multimedia. Barcelona: Gedisa.

COX, Andrew; YEADON, Jane (2002). "Estrategias prácticas en gestión de contenidos”, en El profesional de la información, n6, vol.11, pp. 466-474 http://dx.doi.org/10.1076/epri.11.6.466.15462

ECHEVERRI, Ana Lucía (1995): Recursos Humanos en la empresa informativa. Salamanca: Universidad Pontificia de Salamanca.

EÍTO BRUN, Ricardo (2013). "Madurez de la gestión de contenidos, ¿sinónimo de desgaste o de oportunidades?”, en El Profesional de la Información, n5, vol. 22, pp. 377-380. http://dx.doi.org/10.3145/epi.2013.sep.03

FERNÁNDEZ SANDE, Manuel; PEINADO MIGUEL, Fernando (2012). "La empresa radiofónica actual”, en Sintonizando el futuro: radio y producción sonora en el siglo XXI. Madrid: Instituto RTVE. pp. 29-61

FERNÁNDEZ SANDE, Manuel; RODRÍGUEZ BARBA, Dolores y RODRÍGUEZ PALLARES, Miriam (2012). "La gestión de contenidos como actividad estratégica en empresas de radiodifusión. Estudio de casos en la radio comercial española”, en El profesional de la información, vol. 22 n ${ }^{\circ}$, pp. 392-298

GALLEGO PÉREZ, J. Ignacio (2012). "La audiencia en la radio: viejos roles, nuevas funciones”, en Sintonizando el futuro: radio y producción sonora en el siglo XXI. Madrid: Instituto RTVE. pp. 209-227

GARCÍA LASTRA, José María (2012). "Del modelo productivo de la era analógica al de la radio multicanal”, en Sintonizando el futuro: Radio y producción sonora en el siglo XXI. Madrid: Instituto RTVE.

GONZÁLEZ ALDEA, Patricia (2011). "La radio en Internet: las webs de las cadenas analógicas tradicionales", en Radio 3.0. Una nueva radio para una nueva era. Madrid: Fragua. pp. 89-122.

GONZÁLEZ CONDE, Julia (2010). "La ciberradio. Nueva alternativa de futuro para la radio", en Revista de Estudios de Juventud 88, pp. 51-62.

LÓPEZ VIDALES, Nereida (2011). "La radio se transforma: nuevas tecnologías, nuevos hábitos y nuevos perfiles para el medio más cercano”, en Radio 3.0. Una nueva radio para una nueva era. Madrid: Fragua. pp. 15-40 
LÓPEZ VIDALES, Nereida y ORTIZ SOBRINO, Miguel Ángel (2011). "Viejas nomenclaturas, nuevas competencias. Perfiles profesionales en la radio española”, en Telos: Revista de pensamiento sobre tecnología y sociedad, 87, pp. 63-72.

MARCOS RECIO, Juan Carlos (2014). “Otra forma de plantear la información y documentación en los medios digitales/Another way to make information and documentation in digital media”, en Revista General De Información y

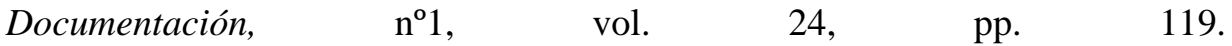
http://dx.doi.org/10.5209/rev_RGID.2014.v24.n1.45467

MARTÍNEZ COSTA, María Pilar (2011). "La nueva organización de la producción”, en Radio 3.0. Una nueva radio para una nueva era. Madrid: Fragua. pp. 123-140.

MATEO, Rosario de; BERGÉS, Laura; SABATER, Marta (2009). Gestión de empresas de comunicación. Sevilla: Comunicación Social.

MICÓ-SANZ, Josep-Lluis, MASIP-MASIP, Pere, y GARCÍA-AVILÉS, José Alberto (2008). "Periodistas que ejercen de documentalistas (¿y viceversa?). Nuevas relaciones entre la redacción y el archivo tras la digitalización de los medios”, en El Profesional de la Información, n³, vol. 18 pp. 284-290.

MINISTERIO DE EDUCACIÓN, CULTURA Y DEPORTE. Definición de la propiedad intelectual. Disponible en: www.mecd.gob.es/cultura-mecd/areascultura/propiedadintelectual/la-propiedad-intelectual/definicion.html >. [23/03/2015]

ORTIZ SOBRINO, Miguel Ángel (2013). "Profesionales polivalentes para la radio y la televisión”, en Perfiles profesionales y espacios de empleo en Información y Comunicación. Madrid: Icono14 pp. 71-93

PÉREZ MONTORO, Mario (2005). "Sistemas de gestión de contenidos en la gestión del conocimiento", en BiD: Textos Universitaris De Biblioteconomia i Documentació. Disponible en: http://bid.ub.edu/14monto2.htm [Consulta: 31/10/14]

PÉREZ SERRANO, María José (2006). La concentración de medios en España: Análisis de casos relevantes en radio, prensa y televisión. Madrid: Servicio de Publicaciones UCM.

RTVE.ES (2013). Disponible en:www.rtve.es [consulta: 18/03/13]

RUBIO LACOBA, María (2005). "El perfil triangular del documentalista digital: Novedades y constantes del emisor de documentación en el periodismo digital”. Scire: Representación y Organización del Conocimiento, 2, (11), pp. 17-32.

RODRÍGUEZ PALLARES, Miriam; RODRÍGUEZ BARBA, Dolores y FERNÁNDEZ SANDE, Manuel (2014). “Activos intangibles en la radio generalista española: La gestión de contenidos y el desarrollo de nuevos perfiles profesionales”, en Miscelánea sobre el entorno audiovisual en 2014. Madrid: Fragua, pp. 97-117 
SALAVERRÍA, Ramón; NEGREDO, Samuel, y PIQUÉ, Antoni María (2008). Periodismo integrado: Convergencia de medios y reorganización de redacciones. Barcelona: Sol90.

SÁNCHEZ GARCÍA, Pilar; CAMPOS DOMÍNGUEZ, Eva y BERROCAL GONZALO, Salomé (2015). "Las funciones inalterables del periodista ante los perfiles multimedia emergentes”. Revista Latina de Comunicación Social, 70, pp. 187-208. Disponible en:www.revistalatinacs.org/070/paper/1042va/ 12es.html [Consulta: 25/03/2015]

SÁNCHEZ TABERNERO, Alfonso (1991). "Publicidad y concentración informativa en Europa”, en Comunicación y Sociedad, 1 y 2.

STAKE, Robert E. (2007). Investigación con estudio de casos. Madrid: Morata. 